\title{
Cardiovascular Safety of Evolocumab: a Systematic Review and Meta-Analysis
}

\author{
Chayakrit Krittanawong ${ }^{1,2} \cdot$ Takeshi Kitai $^{2} \cdot$ HongJu Zhang ${ }^{3} \cdot$ Tao Sun $^{3}$
}

Published online: 10 November 2016

(C) Springer Science+Business Media New York 2016

\section{To the Editor}

We were interested in the Editorial article written by Evan A. Stein [1]. The author reviewed the potential benefit of a proprotein convertase subtilisin/kexin type 9 (PCSK9) inhibitors, alirocumab and evolocumab, in patients with familial hypercholesterolemia $(\mathrm{FH})$ and concluded that PCSK9 inhibitors, lowering low-density lipoprotein cholesterol (LDL-C), should drastically reduce the early mortality and morbidity in $\mathrm{FH}$ patients. However, to date, the cardiovascular safety of evolocumab remains inconclusive. Thus, we performed a systematic review and meta-analysis of all studies to determine the cardiovascular safety of evolocumab use.

We conducted a comprehensive search of MEDLINE, MEDLINE In-Process \& Other Non-Indexed Citations, EMBASE, Scopus, and the Cochrane Central Register of Controlled Trials from database inception through

Chayakrit Krittanawong

Chayakrit.Krittanawong@mountsinai.org

1 Department of Internal Medicine, Icahn School of Medicine at Mount Sinai St' Luke and Mount Sinai West, Tenth Avenue, Suite 3A-09, New York, NY 10019, USA

2 Department of Cardiovascular Medicine, Heart and Vascular Institute, Cleveland Clinic, Cleveland, OH, USA

3 Division of Cardiovascular Disease, Department of Medicine, Mayo Clinic, Rochester, MN, USA
April 2016. Using the DerSimonian and Laird random effects models, we calculated pooled risk ratios (RRs) with $95 \%$ confidence intervals (95\% CIs). The between-study heterogeneity was quantified using the $Q$ statistic and $I^{2}$.

From the results, the pooled analysis demonstrated that evolocumab significantly reduced the risk of cardiovascular events. (RR, 0.53; $95 \% \mathrm{CI}, 0.38$ to 0.74 ; $\left.P<0.001, I^{2}=0 \%, P_{\text {heterogeneity }}=0.498\right)$. It has been known that the reduction of LDL-C can reduce cardiovascular events [2]. LDL-C is associated with inflammation and plaque transformation which leads to atherosclerosis. In the MENDEL-2 (Monoclonal Antibody Against PCSK9 to Reduce Elevated LDL-C in Subjects Currently Not Receiving Drug Therapy for Easing Lipid Levels-2) trial [3], evolocumab yielded $>50 \%$ LDL-C reductions in adults with hypercholesterolemia compared with placebo or ezetimibe. Similarly, RUTHERFORD-2 trial [4], evolocumab yielded $60 \%$ LDL-C reductions compared with placebo. In addition, in the Durable Effect of PCSK9 Antibody Compared with Placebo Study (DESCARTES) trial [5], evolocumab yielded $57 \%$ LDL-C reductions at 52 weeks as compared with placebo.

In conclusion, the results of this meta-analysis showed that evolocumab significantly reduced the risk of cardiovascular events via the mechanism of LDL-C reductions and thereby strengthened the hypothesis of the author. The significance of this meta-analysis is that it shows that in FH patients who cannot tolerate statins, evolocumab is a valuable option for reduction of cardiovascular events. 


\section{Compliance with Ethical Standards}

\section{Funding None.}

Conflict of Interest All authors have no conflict of interest

Ethical Approval This article does not contain any studies with human participants or animals performed by any of the authors.

\section{References}

1. Stein EA. PCSK9: the critical role of familial hypercholesterolemia from discovery to benefit for all. Cardiovasc Drugs Ther. 2016;30: $427-31$.
2. Stone NJ, Robinson JG, Lichtenstein AH, et al. 2013 ACC/ AHA guideline on the treatment of blood cholesterol to reduce atherosclerotic cardiovascular risk in adultsa report of the american college of cardiology/american heart association task force on practice guidelines. J Am Coll Cardiol. 2014;63(25 Pt B):2889-934.

3. Koren MJ, Lundqvist P, Bolognese M, et al. Anti-PCSK9 monotherapy for hypercholesterolemia: the mendel-2 randomized, controlled phase III clinical trial of evolocumab. J Am Coll Cardiol. 2014;63(23):2531-40.

4. Raal FJ, Stein EA, Dufour R, et al. PCSK9 inhibition with evolocumab (AMG 145) in heterozygous familial hypercholesterolaemia (RUTHERFORD-2): a randomised, doubleblind, placebo-controlled trial. Lancet. 2015;385(9965):33140.

5. Blom DJ, Hala T, Bolognese M, et al. A 52-week placebo-controlled trial of evolocumab in hyperlipidemia. N Engl J Med. 2014;370(19): 1809-19. 